\title{
Cancer risk and social inequalities in Italy
}

\author{
Fabrizio Faggiano, Roberto Zanetti, Giuseppe Costa
}

\begin{abstract}
Study objective - To investigate social differences in cancer incidence in Turin, Italy in 1985-87.

Design - A cancer incidence follow up study of the Turin population in relation to socioeconomic characteristics was performed through record linkage between the 1981 census and the cancer registry. $A$ case-control study nested in the cohort was analysed, where cases were subjects with a new diagnosis of cancer in 1985-87 and controls were a sample of the Turin population, frequency matched by sex and age group. Incidence odd ratios (ORs) were calculated for social classes (defined by education, housing tenure, and socioeconomic group) using a logistic regression model.
\end{abstract}

Setting - The study population comprised subjects included in the 1981 Turin census ( $n \cong 1100000)$ who were still alive, 20-69 years old, and were resident in Turin in the middle of study period.

Participants - The analyses were based on 4215 male and 3451 female cases, and on 16913 male and 13838 female controls.

Main results - Compared with the highest educational level, the men in the lowest one showed an OR>2 for respiratory cancers; $O R=1.48$ for stomach cancer; and ORs $<0.7$ for skin, colorectal, and prostate cancers. Women with a primary school education were protected against colorectal $(O R=0.71)$, skin $(O R=0.59)$, and breast cancer $(O R=0 \cdot 66)$ compared with university degree women, but were at risk for cancer of the cervix $(O R=2 \cdot 33)$ and stomach cancer $(O R=2 \cdot 84)$. The association between educational level (primary school $v$ university) and lung cancer risk is negative for men $(O R=2 \cdot 47)$ and positive for women $(O R=0 \cdot 62)$, while the association with housing tenure is negative for both sexes $(O R=1 \cdot 44)$.

Department of Hygiene and Community Medicine, University of Turin, Via Santena 5bis, 10126 Turin, Italy F Faggiano

Epidemiology Unit, LSP, USL TO I, Turin, Italy

G Costa

Piedmont Cancer Registry, Turin, Italy R Zanetti

Correspondence to: Dr F Faggiano.

Accepted for publication February 1994
Studies on the social distribution of cancer occurrence are generally based on mortality.
The cancer sites whose mortality presents a more regular negative correlation with socioeconomic status are stomach, larynx, and lung for men and cervix/uterus for women. Large bowel, breast, ovary, and testis cancers, on the contrary, show a positive gradient, but with less regularity. ${ }^{1}$ These differentials, even though they vary in size, are evident and consistent at any latitude, both in rich countries such as Switzerland or New Zealand ${ }^{2-7}$ and in poorer ones such as Argentina and Brazil. ${ }^{89}$ In Italy, the mortality data from the Turin Longitudinal Study show odds ratios (ORs) for all cancers of 1.2 for males under 60 and 1.2 for males aged $60-74$ between the highest and the lowest educational level. ${ }^{10}$ International reports on social inequalities in cancer incidence are few. ${ }^{11-14}$ More systematic analysis comes from the OPCS Longitudinal Study ${ }^{15}$ and shows a similar distribution of positive and negative associations with social class and mortality.

Social class has also been studied as a prognostic factor in cancer survival. Lower socioeconomic groups usually have poorer survival than higher ones, ${ }^{1617}$ but English and Swedish data indicate that class differences in cancer mortality are primarily affected by differences in incidence rather than in survival. On the other hand, the available evidence is insufficient to identify more than a few of the risk factors such as smoking and alcohol, that link cancer risk to socioeconomic disadvantage, and in any case the relative estimates of the attributable fraction seem to be small.

This paper aimed to describe the social distribution of the incidence of the most relevant cancer sites in Turin, a large industrial town in the north-west of Italy.

\section{Methods}

The results of this work are based on record linkage between the cancer registry (Registro Tumori Piemonte, RTP) and the census data of the Turin population (Studio Longitudinale Torinese, SLT). The cancer incidence data are up to the quality standards defined by the International Agency for Research on Cancer, Lyon, and the census, carried out every 10 years by the National Institute of Statistics, is considered a reliable source for social variables. The SLT included all people living in the City of Turin on the census day (October 21, 1981), followed up to 1989 for mortality and migration. A total of $95 \%$ of the census records matched the corresponding population register record..$^{18}$ Errors in filling in the date of birth in the census questionnaires explain more than $80 \%$ of the failures in record linkage. People who migrated after the census date were considered lost to follow up at the date of migration, while those who were born or 
immigrated in the years between the two censuses are present in the study only at the time of the next census. Therefore, between the censuses, SLT takes the shape of a closed cohort. The RTP provides the cancer incidence data for the Turin population from $1985 .{ }^{19}$ The 1985-87 data were available for the study.

RTP and SLT records were matched according to a key including first name, surname, sex, and date of birth. For the ages included in the study (20-69), 95\% of the male cases and $94.2 \%$ of the female ones were matched. Unsuccessful pairing was mainly due to subjects who had moved to Turin after the 1981 census and were therefore excluded from the SLT. Paired and unpaired cases were compared to determine any selection characteristics: no significant differences were found for either ICD or age.

A case-control design, nested into the cohort, was chosen to study the relationship between the risk of cancer and social class indicators. Four controls for each incident case were randomly selected from the SLT file, using a frequency matching strategy, by sex and five-year age class, without replacement. The controls were: not included in the cancer registry, alive, and residents in Torino halfway through the study period (June 30,1986 ), as determined by the historical city population register. The study is therefore based on 4215 male cases and 16913 controls, and 3451 female cases and 13838 controls. Using the available census data, the study population was classified according to the following socioeconomic indicators: educational levels (university (corresponding to not less than 16 years of school education), high school (12-13 years), middle school (8 years), primary school, or less (0-5 years)); housing tenure; and socioeconomic status, obtained on the basis of the professional position and occupational status. ${ }^{20}$ In this last classification, housewives were considered a separate category because of their specific social condition.

Seventy one per cent of cases could be classified in terms of socioeconomic status by information from the census record. Twenty nine per cent of cases were not classified according to the individual census record: $77 \%$ of them $(22.6 \%$ of the total) were classified according to the census record of the head of family, matched to the individual record through a family identificator code. Information was not available ultimately for $6.7 \%$ of the cases. For controls, the above mentioned percentages were respectively: $72 \cdot 1 \%, 21 \cdot 7 \%$, and $6 \cdot 5 \%$. Education and housing tenure indicators come from the individual record exclusively. The proportions of cases with missing or unhelpful data for these indicators were $0.0 \%$ for education and $2 \cdot 4 \%$ for housing tenure; the corresponding percentages for controls were $0.2 \%$ and $3 \cdot 2 \%$. The distribution of cases and controls entered in the analysis in relation to the social indicators can be found in the first row (labelled 'all sites') of the tables of results.

ORs of incidence have been estimated through a non-conditional logistic model. ${ }^{21}$ Confidence intervals have been calculated at
$95 \%$. The reference group $(O R=1)$ is always represented by the highest social group - university graduates, house owners, managers and professional people. For women, since this reference group is often too small, the two highest categories of the social scale have been pooled to form a reference group (university degree plus high school education; managers plus clerks). To test the trend significance for education, this last indicator was introduced in the model as a continuous variable, on the hypothesis of the equidistance of the different school levels. The results of this analysis have been presented as a $p$ value which stands for the probability associated with the values of $\chi^{2}$ related to the variable in the model. Adjustment for the residual confounding for age was obtained by including in the models the dummy variables related to the same age groups from which the sample had been obtained. The confounding due to birth areas was controlled for with a variable at six levels: province of Turin, the rest of the north-west, the north-east, the centre, the south and the isles of Italy, and foreign countries. It is well known that death rates and cancer incidence are lower in southern than in northern Italian regions ${ }^{223}$ - in some cancer sites they may be even halved - and that there was massive immigration into Turin from the south of Italy in the 50 s and $60 \mathrm{~s}$.

To avoid multicollinearity among indicators, the results presented refer to two different models in which the variables analysed are as follows: model 1 - education and housing tenure, standing respectively for the cultural level and for the income; model 2 - socioeconomic status, and representing a summary measure for prestige, income. ${ }^{24}$ Analysis was performed for all cancers and for some sites chosen because of the number of observations. The distribution of cases by site is reported in first column of table 1 for men and of table 3 for women. Larynx and bladder cancers for women did not have sufficient number of cases for the analysis ( $n=7$ and $n=64$ respectively). Skin cancer refers only to non-melanoma cancers (ICD 173); malignant melonoma was excluded because of the small numbers $(M=61 ; F=66)$.

\section{Results}

In men the lower educational group shows a $15 \%$ significant, excess risk for all cancer sites in each class indicator (tables 1 and 2). For women the observed differentials are not consistent between the three indicators: the risk of cancer is lower for less educated women and for those in the lower socioeconomic group and is higher among housing tenants (tables 3 and 4). These differentials are the result of sitespecific associations of different direction and size.

\section{MEN: NEGATIVE ASSOCIATIONS WITH SOCIAL} CLASS

Upper respiratory and digestive tract, larynx, stomach, and lung cancers present a consistent negative trend along the three social scales, with trends for education and risk for lower socioeconomic groups always significant, ex- 
Table 1 Differences in men's risk of cancer in relation to education and housing tenure in Turin in the 1980s. Absolute numbers (cases/controls), odds ratios (OR), 95\% confidence intervals (CI), p value for trend in education are given. (ORs are adjusted for age, birth area, and variables presented in table.)

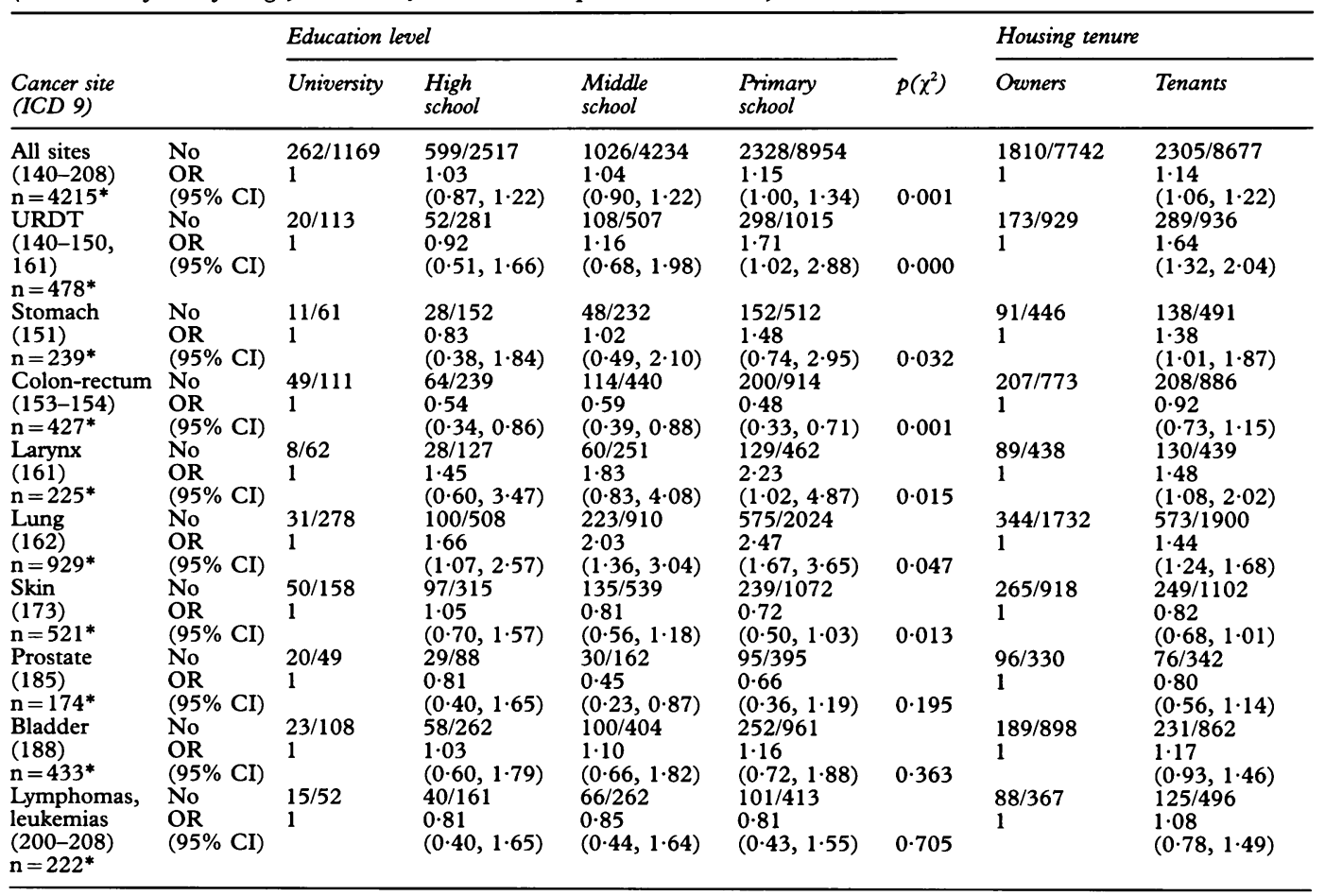

*Site specific number of cases. URDT $=$ cancers of the upper respiratory and digestive tract.

Table 2 Differences in men's risk of cancer in relation to socioeconomic groups in Turin in the 1980s. Absolute numbers (cases/controls), odds ratios (OR), and 95\% confidence intervals (CI) are given. (ORs are adjusted for age and birth area.)

\begin{tabular}{|c|c|c|c|c|c|c|}
\hline \multirow[b]{2}{*}{$\begin{array}{l}\text { Cancer site } \\
\text { (ICD 9) }\end{array}$} & & \multicolumn{5}{|c|}{ Socioeconomic group } \\
\hline & & $\begin{array}{l}\text { Managers/ } \\
\text { professionals }\end{array}$ & Clerks & $\begin{array}{l}\text { Self } \\
\text { employed }\end{array}$ & $\begin{array}{l}\text { Manual } \\
\text { workers }\end{array}$ & Unemployed \\
\hline $\begin{array}{l}\text { All sites } \\
(140-208)\end{array}$ & $\begin{array}{l}\text { No } \\
\text { OR } \\
(95 \% \mathrm{CI})\end{array}$ & $\begin{array}{l}478 / 2021 \\
1\end{array}$ & $\begin{array}{l}793 / 3462 \\
0 \cdot 97 \\
(0 \cdot 86,1 \cdot 10)\end{array}$ & $\begin{array}{l}496 / 2022 \\
1.05 \\
(0 \cdot 91,1 \cdot 21)\end{array}$ & $\begin{array}{l}2031 / 7857 \\
1 \cdot 14 \\
(1 \cdot 01,1 \cdot 28)\end{array}$ & $\begin{array}{l}67 / 232 \\
1 \cdot 26 \\
(0 \cdot 94,1 \cdot 68)\end{array}$ \\
\hline URDT & No & $30 / 209$ & $77 / 404$ & $56 / 270$ & $261 / 870$ & $13 / 23$ \\
\hline$(140-150,161)$ & $\begin{array}{l}\text { OR } \\
(95 \% \mathrm{CI})\end{array}$ & 1 & $\begin{array}{l}1 \cdot 37 \\
(0 \cdot 87,2 \cdot 16)\end{array}$ & $\begin{array}{l}1.53 \\
(0.94,2.48)\end{array}$ & $\begin{array}{l}2 \cdot 51 \\
(1.65,3 \cdot 81)\end{array}$ & $\begin{array}{l}4 \cdot 61 \\
(2 \cdot 08,10 \cdot 18)\end{array}$ \\
\hline Stomach & No & $24 / 117$ & $40 / 197$ & $24 / 100$ & $123 / 460$ & $3 / 14$ \\
\hline (151) & $\begin{array}{l}\text { OR } \\
(95 \% \mathrm{CI})\end{array}$ & 7040 & $\begin{array}{l}0.99 \\
(0.57,1.72)\end{array}$ & $\begin{array}{l}1 \cdot 19 \\
(0 \cdot 63,2 \cdot 23)\end{array}$ & $\begin{array}{l}1 \cdot 30 \\
(0 \cdot 79,2 \cdot 14)\end{array}$ & $\begin{array}{l}1 \cdot 00 \\
(0 \cdot 27,3 \cdot 77)\end{array}$ \\
\hline $\begin{array}{l}\text { Colon-rectum } \\
(153-154)\end{array}$ & $\begin{array}{l}\text { No } \\
\text { OR } \\
(95 \% \mathrm{CI})\end{array}$ & $\begin{array}{l}70 / 196 \\
1\end{array}$ & $\begin{array}{l}86 / 357 \\
0 \cdot 69 \\
(0 \cdot 48,0.99)\end{array}$ & $\begin{array}{l}49 / 194 \\
0.72 \\
(0.47,1.99)\end{array}$ & $\begin{array}{l}173 / 823 \\
0.63 \\
(0 \cdot 45,0.87)\end{array}$ & $\begin{array}{l}8 / 11 \\
2 \cdot 11 \\
(0 \cdot 81,5 \cdot 50)\end{array}$ \\
\hline Larynx & No & $17 / 105$ & $43 / 182$ & $30 / 132$ & $119 / 414$ & $\begin{array}{l}4.81,5.50) \\
4 / 10\end{array}$ \\
\hline$(161)$ & $\begin{array}{l}\text { OR } \\
(95 \% \mathrm{CI})\end{array}$ & & $\begin{array}{l}1.59 \\
(0.86,2.93)\end{array}$ & $\begin{array}{l}1.57 \\
(0.82,3.01)\end{array}$ & $\begin{array}{l}2 \cdot 14 \\
(1 \cdot 21,3 \cdot 79)\end{array}$ & $\begin{array}{l}2.95 \\
(0.81,10.71)\end{array}$ \\
\hline $\begin{array}{l}\text { Lung } \\
(162)\end{array}$ & $\begin{array}{l}\text { No } \\
\text { OR } \\
(95 \% \mathrm{CI})\end{array}$ & $\begin{array}{l}71 / 451 \\
1\end{array}$ & $\begin{array}{l}154 / 755 \\
1 \cdot 30 \\
(0 \cdot 96,1 \cdot 76)\end{array}$ & $\begin{array}{l}124 / 437 \\
1 \cdot 80 \\
(1 \cdot 31,2 \cdot 49)\end{array}$ & $\begin{array}{l}487 / 1759 \\
1 \cdot 81 \\
(1 \cdot 37,2 \cdot 38)\end{array}$ & $\begin{array}{l}11 / 56 \\
1 \cdot 32 \\
(0 \cdot 65,2 \cdot 66)\end{array}$ \\
\hline $\begin{array}{l}\text { Skin } \\
\text { (173) }\end{array}$ & $\begin{array}{l}\text { No } \\
\text { OR } \\
(95 \% \mathrm{CI})\end{array}$ & $\begin{array}{l}93 / 251 \\
1\end{array}$ & $\begin{array}{l}122 / 435 \\
0 \cdot 75 \\
(0.55,1.03)\end{array}$ & $\begin{array}{l}49 / 239 \\
0.55 \\
(0 \cdot 37,0 \cdot 81)\end{array}$ & $\begin{array}{l}212 / 956 \\
0.59 \\
(0.44,0.78)\end{array}$ & $\begin{array}{l}5 / 24 \\
0 \cdot 55 \\
(0 \cdot 20,1 \cdot 50)\end{array}$ \\
\hline $\begin{array}{l}\text { Prostate } \\
\text { (185) }\end{array}$ & $\begin{array}{l}\text { No } \\
\text { OR } \\
(95 \% \mathrm{CI})\end{array}$ & $\begin{array}{l}21 / 70 \\
1\end{array}$ & $\begin{array}{l}32 / 149 \\
0 \cdot 73 \\
(0 \cdot 39,1 \cdot 36)\end{array}$ & $\begin{array}{l}26 / 78 \\
1 \cdot 17 \\
(0 \cdot 60,2 \cdot 28)\end{array}$ & $\begin{array}{l}76 / 295 \\
0.94 \\
(0.53,1.66)\end{array}$ & $\begin{array}{l}1 / 5 \\
0 \cdot 75 \\
(0 \cdot 08,6 \cdot 92)\end{array}$ \\
\hline $\begin{array}{l}\text { Bladder } \\
\text { (188) }\end{array}$ & $\begin{array}{l}\text { No } \\
\text { OR } \\
(95 \% \mathrm{CI})\end{array}$ & $\begin{array}{l}54 / 196 \\
1\end{array}$ & $\begin{array}{l}75 / 349 \\
0 \cdot 79 \\
(0 \cdot 53,1 \cdot 17)\end{array}$ & $\begin{array}{l}57 / 211 \\
0.99 \\
(0 \cdot 65,1.51)\end{array}$ & $\begin{array}{l}214 / 810 \\
0 \cdot 98 \\
(0 \cdot 69,1 \cdot 39)\end{array}$ & $\begin{array}{l}4 / 15 \\
0.98 \\
(0.31,3.08)\end{array}$ \\
\hline $\begin{array}{l}\text { Lymphomas, } \\
\text { leukemias } \\
(200-208)\end{array}$ & $\begin{array}{l}\text { No } \\
\text { OR } \\
(95 \% \mathrm{CI})\end{array}$ & $\begin{array}{l}29 / 98 \\
1\end{array}$ & $\begin{array}{l}51 / 186 \\
0.94 \\
(0.56,1.58)\end{array}$ & $\begin{array}{l}26 / 104 \\
0 \cdot 85 \\
(0 \cdot 47,1 \cdot 56)\end{array}$ & $\begin{array}{l}100 / 419 \\
0 \cdot 85 \\
(0 \cdot 52,1 \cdot 39)\end{array}$ & $\begin{array}{l}6 / 27 \\
0 \cdot 77 \\
(0 \cdot 28,2 \cdot 12)\end{array}$ \\
\hline
\end{tabular}

cept for the stomach cancer along the socioeconomic scale. Bladder cancer shows a slight negative non-significant association with education and housing tenure.

MEN: POSITIVE ASSOCIATIONS WITH SOCIAL

CLASS

Less educated people are significantly protected against colorectal and skin cancer. Man- agers and professional people seem to be the only socioeconomic group at high risk for these sites. Prostate cancer shows a slight, non-significant positive association with education and housing tenure.

MEN: SITES PRESENTING NO ASSOCIATION WITH SOCIAL CLASS

Lymphoma and leukaemia do not seem to have 
Table 3 Social differences in the women's risk of cancer in relation to education and housing tenure in Turin in the 1980s. Absolute numbers (cases/controls), odds ratios (OR), 95\% confidence intervals (CI), and p value for trend are given. (ORs are adjusted for age, birth area, and variables presented in table.)

\begin{tabular}{|c|c|c|c|c|c|c|c|c|}
\hline \multirow[b]{2}{*}{$\begin{array}{l}\text { Cancer site } \\
\text { (ICD 9) }\end{array}$} & & \multicolumn{4}{|c|}{ Education level } & \multirow[b]{2}{*}{$p\left(\chi^{2}\right)$} & \multicolumn{2}{|c|}{ Housing tenure } \\
\hline & & University & $\begin{array}{l}\text { High } \\
\text { school }\end{array}$ & $\begin{array}{l}\text { Middle } \\
\text { school }\end{array}$ & $\begin{array}{l}\text { Primary } \\
\text { school }\end{array}$ & & Owners & Tenants \\
\hline 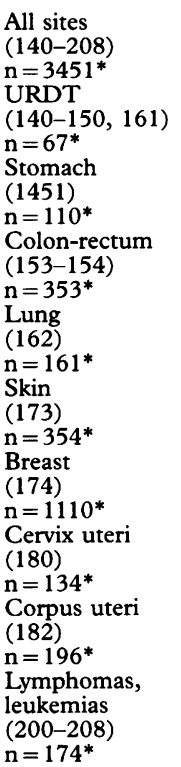 & $\begin{array}{l}\text { No } \\
\text { OR } \\
(95 \% \text { CI) } \\
\text { No } \\
\text { OR } \\
(95 \% \text { CI) } \\
\text { No } \\
\text { OR } \\
(95 \% \text { CI) } \\
\text { No } \\
\text { OR } \\
(95 \% \text { CI) } \\
\text { No } \\
\text { OR } \\
(95 \% \text { CI) } \\
\text { No } \\
\text { OR } \\
(95 \% \text { CI) } \\
\text { No } \\
\text { OR } \\
(95 \% \text { CI) } \\
\text { No } \\
\text { OR } \\
(95 \% \text { CI) } \\
\text { No } \\
\text { OR } \\
(95 \% \text { CI) } \\
\text { No } \\
\text { OR } \\
(95 \% \text { CI) }\end{array}$ & $\begin{array}{l}19 / 47 \\
1 \\
44 / 126 \\
1\end{array}$ & $\begin{array}{l}436 / 1681 \\
0 \cdot 87 \\
(0 \cdot 69,1 \cdot 10) \\
7 / 31 \\
1 \\
9 / 67 \\
1 \\
40 / 148 \\
0 \cdot 78 \\
(0 \cdot 38,1 \cdot 58) \\
26 / 81 \\
1 \\
45 / 181 \\
0 \cdot 62 \\
(0 \cdot 32,1 \cdot 17) \\
161 / 551 \\
0 \cdot 86 \\
(0 \cdot 58,1 \cdot 29) \\
10 / 94 \\
1 \\
(0 \cdot 81,3 \cdot 86) \\
28 / 98 \\
1 \\
31 / 109 \\
1\end{array}$ & $\begin{array}{l}942 / 3321 \\
0 \cdot 90 \\
(0 \cdot 73,1 \cdot 12) \\
21 / 59 \\
1 \cdot 19 \\
(0 \cdot 43,3 \cdot 31) \\
22 / 97 \\
2 \cdot 47 \\
(0 \cdot 93,6 \cdot 51) \\
84 / 331 \\
0 \cdot 75 \\
(0 \cdot 40,1 \cdot 42) \\
44 / 162 \\
0 \cdot 74 \\
(0 \cdot 41,1 \cdot 35) \\
97 / 328 \\
0 \cdot 79 \\
(0 \cdot 44,1 \cdot 42) \\
316 / 1085 \\
0 \cdot 84 \\
(0 \cdot 56,1 \cdot 21) \\
32 / 131 \\
1 \cdot 77 \\
(1 \cdot 09,4 \cdot 97) \\
50 / 172 \\
0 \cdot 93 \\
(0 \cdot 54,1 \cdot 60) \\
53 / 188 \\
0 \cdot 92 \\
(0 \cdot 54,1 \cdot 56)\end{array}$ & $\begin{array}{l}1944 / 8410 \\
0 \cdot 76 \\
(0.62,0 \cdot 94) \\
39 / 178 \\
0 \cdot 82 \\
(0 \cdot 30,2 \cdot 24) \\
79 / 279 \\
2 \cdot 84 \\
(1 \cdot 15,7 \cdot 01) \\
214 / 888 \\
0 \cdot 71 \\
(0 \cdot 38,1 \cdot 32) \\
91 / 402 \\
0 \cdot 62 \\
(0 \cdot 35,1 \cdot 11) \\
193 / 858 \\
0 \cdot 59 \\
(0 \cdot 33,1 \cdot 04) \\
589 / 2684 \\
0 \cdot 66 \\
(0 \cdot 46,0 \cdot 96) \\
92 / 313 \\
2 \cdot 33 \\
0 \cdot 012 \\
118 / 515 \\
0 \cdot 81 \\
(0 \cdot 49,1 \cdot 34) \\
90 / 400 \\
0 \cdot 72 \\
(0 \cdot 42,1 \cdot 23)\end{array}$ & $\begin{array}{l}0.314 \\
0.099\end{array}$ & $\begin{array}{l}1520 / 6141 \\
1 \\
20 / 120 \\
1 \\
43 / 194 \\
1 \\
162 / 659 \\
1 \\
57 / 279 \\
1 \\
184 / 652 \\
1 \\
503 / 1917 \\
1 \\
34 / 243 \\
1 \\
(1 \cdot 46,3 \cdot 52) \\
100 / 373 \\
1 \\
71 / 289 \\
1\end{array}$ & $\begin{array}{l}1844 / 7260 \\
1 \cdot 06 \\
(0 \cdot 98,1 \cdot 15) \\
46 / 143 \\
1 \cdot 99 \\
(1 \cdot 11,3 \cdot 59) \\
64 / 233 \\
1 \cdot 09 \\
(0 \cdot 70,1 \cdot 71) \\
181 / 707 \\
1 \cdot 01 \\
(0 \cdot 79,1 \cdot 28) \\
99 / 342 \\
1 \cdot 44 \\
(0 \cdot 99,2 \cdot 10) \\
164 / 714 \\
1 \cdot 10 \\
(0 \cdot 70,1 \cdot 72) \\
580 / 2400 \\
0 \cdot 98 \\
(0 \cdot 86,1 \cdot 13) \\
97 / 286 \\
2 \cdot 27 \\
\\
93 / 390 \\
0 \cdot 98 \\
(0 \cdot 70,1 \cdot 35) \\
100 / 393 \\
1 \cdot 03 \\
(0 \cdot 73,1 \cdot 46)\end{array}$ \\
\hline
\end{tabular}

${ }^{*}$ Site specific number of cases.

Table 4 Differences in women's risk of cancer in relation to socioeconomic group. Absolute numbers (cases/controls), odds ratios (OR), 95\% confidence intervals (CI) are given. (ORs are adjusted for age and birth area)

\begin{tabular}{|c|c|c|c|c|c|c|c|}
\hline \multirow[b]{2}{*}{$\begin{array}{l}\text { Cancer site } \\
\text { (ICD 9) }\end{array}$} & & \multicolumn{6}{|c|}{ Socioeconomic group } \\
\hline & & $\begin{array}{l}\text { Managers/ } \\
\text { professionals }\end{array}$ & Clerks & $\begin{array}{l}\text { Self } \\
\text { employed }\end{array}$ & $\begin{array}{l}\text { Manual } \\
\text { workers }\end{array}$ & Housewives & Unemployed \\
\hline $\begin{array}{l}\text { All sites } \\
(140-208)\end{array}$ & $\begin{array}{l}\text { No } \\
\text { OR } \\
(95 \% \mathrm{CI})\end{array}$ & $\begin{array}{l}112 / 375 \\
1\end{array}$ & $\begin{array}{l}489 / 1766 \\
0 \cdot 93 \\
(0 \cdot 74,1 \cdot 18)\end{array}$ & $\begin{array}{l}216 / 928 \\
0 \cdot 80 \\
(0 \cdot 62,1 \cdot 03)\end{array}$ & $\begin{array}{l}451 / 1891 \\
0 \cdot 86 \\
(0 \cdot 68,1 \cdot 09)\end{array}$ & $\begin{array}{l}1971 / 8042 \\
0 \cdot 86 \\
(0 \cdot 69,1 \cdot 07)\end{array}$ & $\begin{array}{l}48 / 184 \\
0.92 \\
(0.62,1.35)\end{array}$ \\
\hline $\begin{array}{l}\text { Stomach } \\
(151)\end{array}$ & $\begin{array}{l}\text { No } \\
\text { OR } \\
(95 \% \text { CI })\end{array}$ & & $\begin{array}{l}13 / 69 \\
1\end{array}$ & $\begin{array}{l}4 / 43 \\
0 \cdot 45 \\
(0 \cdot 14,1.49)\end{array}$ & $\begin{array}{l}22 / 48 \\
2 \cdot 21 \\
(0 \cdot 98,4 \cdot 94)\end{array}$ & $\begin{array}{l}64 / 255 \\
1 \cdot 22 \\
(0 \cdot 61,2 \cdot 44)\end{array}$ & $\begin{array}{l}1 / 10 \\
0.54 \\
(0.06,4 \cdot 66)\end{array}$ \\
\hline $\begin{array}{l}\text { Colon-rectum } \\
(153-154)\end{array}$ & $\begin{array}{l}\text { No } \\
\text { OR } \\
(95 \% \text { CI })\end{array}$ & $\begin{array}{l}12 / 36 \\
1\end{array}$ & $\begin{array}{l}43 / 152 \\
0 \cdot 84 \\
(0 \cdot 40,1 \cdot 75)\end{array}$ & $\begin{array}{l}20 / 91 \\
0 \cdot 67 \\
(0 \cdot 30,1 \cdot 51)\end{array}$ & $\begin{array}{l}35 / 161 \\
0 \cdot 68 \\
(0 \cdot 32,1.45)\end{array}$ & $\begin{array}{l}220 / 891 \\
1 \cdot 33 \\
(0 \cdot 67,2 \cdot 61)\end{array}$ & $\begin{array}{l}4 / 4 \\
3 \cdot 13 \\
(0 \cdot 67,14 \cdot 61)\end{array}$ \\
\hline $\begin{array}{l}\text { Lung } \\
(162)\end{array}$ & $\begin{array}{l}\text { No } \\
\text { OR } \\
(95 \% \text { CI })\end{array}$ & & $\begin{array}{l}34 / 85 \\
1\end{array}$ & $\begin{array}{l}18 / 54 \\
0 \cdot 86 \\
(0 \cdot 44,1 \cdot 71)\end{array}$ & $\begin{array}{l}16 / 80 \\
0 \cdot 45 \\
(0 \cdot 22,0.91)\end{array}$ & $\begin{array}{l}80 / 382 \\
0 \cdot 49 \\
(0 \cdot 30,0 \cdot 82)\end{array}$ & $\begin{array}{l}2 / 10 \\
0.41 \\
(0.08,2.09)\end{array}$ \\
\hline $\begin{array}{l}\text { Skin } \\
(173)\end{array}$ & $\begin{array}{l}\text { No } \\
\text { OR } \\
(95 \% \mathrm{CI})\end{array}$ & $\begin{array}{l}15 / 35 \\
1\end{array}$ & $\begin{array}{l}47 / 172 \\
0 \cdot 63 \\
(0 \cdot 32,1 \cdot 26)\end{array}$ & $\begin{array}{l}25 / 94 \\
0 \cdot 64 \\
(0 \cdot 30,1 \cdot 35)\end{array}$ & $\begin{array}{l}35 / 193 \\
0 \cdot 45 \\
(0 \cdot 22,0 \cdot 92)\end{array}$ & $\begin{array}{l}211 / 825 \\
0 \cdot 63 \\
(0 \cdot 34,1 \cdot 20)\end{array}$ & $\begin{array}{l}3 / 16 \\
0 \cdot 42 \\
(0 \cdot 11,1 \cdot 69)\end{array}$ \\
\hline $\begin{array}{l}\text { Breast } \\
(174)\end{array}$ & $\begin{array}{l}\text { No } \\
\text { OR } \\
(95 \% \mathrm{CI})\end{array}$ & $\begin{array}{l}38 / 114 \\
1\end{array}$ & $\begin{array}{l}187 / 628 \\
0 \cdot 89 \\
(0 \cdot 59,1 \cdot 33)\end{array}$ & $\begin{array}{l}68 / 313 \\
0.67 \\
(0.42,1.05)\end{array}$ & $\begin{array}{l}159 / 665 \\
0.77 \\
(0 \cdot 51,1 \cdot 16)\end{array}$ & $\begin{array}{l}616 / 2515 \\
0 \cdot 77 \\
(0 \cdot 52,1 \cdot 12)\end{array}$ & $\begin{array}{l}8 / 53 \\
0 \cdot 48 \\
(0 \cdot 21,1 \cdot 09)\end{array}$ \\
\hline $\begin{array}{l}\text { Cervix uteri } \\
(180)\end{array}$ & $\begin{array}{l}\text { No } \\
\text { OR } \\
(95 \% \text { CI })\end{array}$ & & $l_{1}^{16 / 95}$ & $\begin{array}{l}11 / 37 \\
1 \cdot 88 \\
(0 \cdot 79,4 \cdot 48)\end{array}$ & $\begin{array}{l}24 / 77 \\
2 \cdot 15 \\
(1 \cdot 03,4 \cdot 47)\end{array}$ & $\begin{array}{l}78 / 292 \\
1 \cdot 81 \\
(0 \cdot 97,3 \cdot 41)\end{array}$ & $\begin{array}{l}3 / 11 \\
1 \cdot 69 \\
(0 \cdot 42,6 \cdot 80)\end{array}$ \\
\hline $\begin{array}{l}\text { Corpus uteri } \\
(182)\end{array}$ & $\begin{array}{l}\text { No } \\
\text { OR } \\
(95 \% \text { CI })\end{array}$ & & $\begin{array}{l}27 / 101 \\
1\end{array}$ & $\begin{array}{l}8 / 42 \\
0 \cdot 73 \\
(0 \cdot 31,1 \cdot 75)\end{array}$ & $\begin{array}{l}34 / 105 \\
1 \cdot 35 \\
(0 \cdot 75,2 \cdot 44)\end{array}$ & $\begin{array}{l}116 / 488 \\
0.93 \\
(0.57,1.51)\end{array}$ & $\begin{array}{l}2 / 8 \\
1 \cdot 03 \\
(0 \cdot 20,5 \cdot 24)\end{array}$ \\
\hline $\begin{array}{l}\text { Lymphomas, } \\
\text { leukemias } \\
(200-208)\end{array}$ & $\begin{array}{l}\text { No } \\
\text { OR } \\
(95 \% \mathrm{CI})\end{array}$ & & $\begin{array}{l}32 / 110 \\
1\end{array}$ & $\begin{array}{l}7 / 46 \\
0 \cdot 52 \\
(0 \cdot 21,1 \cdot 28)\end{array}$ & $\begin{array}{l}19 / 101 \\
0.65 \\
(0.34,1.25)\end{array}$ & $\begin{array}{l}101 / 386 \\
0.90 \\
(0.54,1.48)\end{array}$ & $\begin{array}{l}7 / 22 \\
1 \cdot 11 \\
(0 \cdot 43,2 \cdot 90)\end{array}$ \\
\hline
\end{tabular}

any association with social class, even though lower classes seem to have a slight and constant protection. The separated analysis of lymphoma and leukaemia (results not presented) shows similar risk patterns: $O R=0.90(95 \%$ CI $0.40,2 \cdot 04)$ and $\mathrm{OR}=0.60(95 \%$ CI 0.20 , $1 \cdot 83$ ) for primary education, respectively.

\section{WOMEN: NEGATIVE ASSOCIATIONS WITH SOCIAL}

CLASS

Among women, the only two sites that show consistent negative associations with social class are stomach and cervix. Stomach cancer presents a higher risk for women with primary education than those with a university degree. Cervical cancer shows a significant trend for education and a strong significant excess among tenants. Risks for both stomach and cervix cancer are at least double for manual workers compared with managers. Housewives show an almost significant risk for cancer of the cervix. Upper respiratory and digestive tract cancers show a strongly significant excess among tenants. Because of the small number of cases in the reference category, the model with socioeconomic groups could not be calculated. 
WOMEN: POSITIVE ASSOCIATIONS WITH SOCIAL CLASS

Breast, skin, lung, and large bowel cancers are more frequent in higher social levels. Breast cancer shows a positive and significant trend with education while skin cancer shows a clear but not significant trend for this indicator, and a significant protection for female manual employees. The risks of lung cancer in both manual working women and housewives are halved compared with managers, but the same risk, adjusted for education among tenants, is $44 \%$ higher than for house owners, and is almost significant. Colorectal cancer shows a slight, but not significant, positive association within education and socioeconomic groups.

\section{WOMEN: SITES PRESENTING NO ASSOCIATION} WITH SOCIAL CLASS

Cancer of the uterus, lymphomas, and leukaemias do not show any coherent association with the social scales used in this analysis. Lymphomas, when analysed separately (data not presented), show an OR of 0.57 (95\% CI $0.32,1.04$ ) for less educated women; self employed women and manual workers present ORs of $0.56(95 \% \mathrm{CI} 0.11,1.09)$ and of 0.66 $(95 \%$ CI $0.32,1.35)$ respectively.

\section{Discussion}

There is strong evidence of socioeconomic differences in the risk of specific cancers in Turin in the 1980s. The agreement with published studies ${ }^{1}$ showing that lung, stomach, larynx, and uterus cancers are negatively associated with social class, and large bowel and breast cancers are positively associated, strengthens the validity of the social indicators used in our study. The positive association for skin cancer is a new finding that does not seem to have been reported before. Because skin cancers other than malignant melanoma are clinically benign ${ }^{1}$, this finding could be missed in mortality studies. Our results reflect both a greater tendency of the higher socioeconomic classes to access diagnostic services and an effect of social differences in sun exposure while on vacation, since there is little occupational exposure to the sun in Turin.

Environmental risk factors that make a substantial contribution to cancer are associated with the greatest social differentials in cancer risk. ${ }^{1}$ The main factor responsible for upper respiratory and digestive tract cancers is alcohol and its prevalence distribution in Italy in 1983 was negatively associated with education. ${ }^{25}$ The prevalence odds ratios (PORs) of heavy alcohol consumers in Italy in 1983 (POR $=1.9$ for less educated $v$ more highly educated men and no differences in women) seems to agree with our ORs (OR $=1.71$ for less educated men; no differences in women) for upper respiratory and digestive tract cancers in both sexes. Among men, lung and larynx cancers present a social profile that is consistent with Italian social differentials in smoking prevalence, ${ }^{26}$ but the POR of less educated people $v$ more educated ones was lower (1.35 in 1986). Oc- cupational exposures in an industrialised town like Turin may play an additional, important role in producing differentials in lung cancer. The interaction of alcohol and smoking may explain the deep differences in larynx cancer. The inverse trend of ORs for lung cancer in women reflects the social distribution of smoking in Italy, which ranges from $14.2 \%$ for the lowest to $23.3 \%$ for highest educational level. ${ }^{26}$ Rates of quitting smoking in both sexes are higher in higher social groups (ranging from 24.6 for the less educated to 29.3 for the more educated group): the widening of social negative differentials in lung cancer for males and an inversion of the social positive differential for women may therefore be predicted. The positive social differential in the risk of large bowel cancer could be attributable to the corresponding social profile in dietary habits, especially low fibre and high animal fat consumption. ${ }^{1}$ In fact, in Italy, disadvantaged social groups showed, in a 1984 survey, healthier dietary habits in relation to these nutrients. ${ }^{27}$

The above considerations suggest that an important component of the social differences in cancer risk, can be considered solvable through unbalanced health promotion intervention - that is those targeted at the social categories which are more at risk. A similar conclusion can be drawn for invasive cervical cancer, for which an effective screening test (Pap test) is available: the prevalence of the use of this early diagnosis test in Italy in 1986 was negatively associated with social class. ${ }^{28}$ Such a social distribution is similar to the use of mammographic screening for breast cancer, but in this case it goes in the right direction to protect the social group at highest risk for this cancer.

As has been discussed in studies on inequalites in health, ${ }^{29}$ the issue is more complex. Educational level is not only a social indicator, in many cases it is a real protection factor. Education allows people to recognise the factors affecting health and the best solutions to health problems. Moreover, education, as well as the other class indicators, is considered a strong predictor of social instability, ${ }^{12}$ and it can affect hazardous habits by putting the satisfaction of passing needs below long term expectations. Increasing the level of education in a country, by promoting the school attendance of young people (at national level in $1981,61 \cdot 8 \%$ of the population over 6 years had not accomplished the compulsory education stated by a 1963 law) $)^{30}$ or prolonging compulsory education (in Italy it is up to 14 years), may be a suitable way of intervening to reduce differences and to increase the global health status of the population.

Differences in the risk of lung cancer in women associated with housing tenure and education follow the opposite direction. It seems a good example of the different meanings of two social indicators. Our interpretation is that income, represented by housing tenure, is a determinant of protective smoking habits. On the other hand, education is an independent predictor of the tendency towards emancipation, which has meant that during the last 
20 years women have followed the smoking habits of men. The socioeconomic group is an indicator that is more sensitive to education than to income. The lower number of women in the manager category $(\mathrm{ca} / \mathrm{co}=7 / 13)$ makes the income differences between reference category (managers plus clerks) and manual employed workers very small.

This study was partially supported by a grant from AIRC (Italian Association for Research on Cancer). Data processing facilities supported by CSI-Piemonte (Consorzio per il Sistema Insupported by CSI-Piemonte (Consor

We thank Franco Gogliani and Piera Vicari for technical We thank Franco Gogliani and Piera Vicari for technical
support and Moreno Demaria for essential advice on data processing.

1 Tomatis L, ed. Cancer: causes, occurrence and control. Lyon IARC, 1990. IARC Scientific Publications no 100.

2 Goldblatt P, ed. Longitudinal Study: mortality and social organisation. London: HMSO, 1990.

3 Marmot MG, Smith GD, Stanfield S, et al. Health in equalities among British civil servants. Lancet 1991;337 1387-93.

4 Smith GD, Leon D, Shipley M, et al. Socioeconomic differentials in cancer among men. Int 7 Epidemiol 1991;20: 339-345.

5 Fox AJ, Goldblatt P, Jones DR. Social class mortality differentials: artefact, selection or life circumstances?. $\mathcal{F} \mathrm{Ep}$ idemiol Community Health 1985;39:1-8.

6 Levi F, Negri E, La Vecchia C, et al. Socioeconomic groups and cancer risk at death in the Swiss canton of Vaud. Int and cancer risk at death in the

7 Pearce NE, Howard JK. Occupation, social class and male cancer mortality in New Zealand. Int $\mathcal{7}$ Epidemiol 1986 15:456-62.

8 Poletto L, Morini JC. Cancer mortality and some socioeconomic correlates in Rosario, Argentina. Cancer Lett 1990;49:201-5.

9 Rumel D. Razoes de mortalidade frente ao efeito desigualdade em estudos de mortalidade associada a categorias ocupacionais e niveis sociais. Rev Saude Publica 1988;22:335-40.

10 Faggiano F, Costa G. Classe sociale e mortalità a Torino negli anni '80. Polis 1990;IV:471-96.

11 Devesa S, Diamond EL. Socioeconomic and racial differences in lung cancer incidence. Am $\mathcal{F}$ Epidemiol 1983;118 818-31.
12 Greenberg RS, Haber MJ, Scott Clark W, et al. The relation of socioeconomic status to oral and pharyngeal cancer. Epidemiology 1991;2:194-200.

13 Alexander FE, Ricketts TJ, McKinney PA, et al. Community lifestyle characteristics and incidence of Hodgkin's disease in young people. Int 7 Cancer 1991;48:10-14.

14 Alexander FE, Cartwright RA, McKinney PA, et al. Leukemia incidence, social class and estuaries: an ecological analysis. F Public Health Med 1990;12:109-117.

15 Leon D. The social distribution of cancer. London: HMSO, 1988 Longitudinal Study 5, Series LS no 3.

16 Kogevinas M, Marmot MG, Fox AJ, Golblatt PO. Socioeconomic differences in cancer survival. $\mathcal{f}$ Epidemiol Community Health 1991;45:216-19.

17 Vagero D, Persson G. Cancer survival and social class in Sweden. F Epidemiol Community Health 1987;41:204-9.

18 Costa G, Demaria M. Un sistema longitudinale di sorveglianza della mortalità secondo le caratteristiche socioeconomiche, come rilevate ai censimenti di popolazione: economiche come e prevenzione 1988;36:37-47.

19 Zanetti R, Crosignani P, eds. Cancer in Italy. Torino: Lega per la Lotta Contro i Tumori, Associazione Italiana di per la Lotta Contro

20 Sylos Labini P. Le classi sociali negli anni '80. Bari: Laterza, 1986.

21 Breslow NE, Day NE. Statistical methods in cancer research. Vol 1. The analysis of case-control studies. Lyon: IARC, 1990. IARC Scientific Publications no 32

22 Capocaccia R, Farchi S, Mariotti A, et al. La mortalita in Italia nell'anno 1987. Roma: Rapporti ISTISAN 1990. 90/ 18.

23 Costa G. Zanetti $\mathrm{R}$, Bena $\mathrm{A}$, et al. La mortalità secondo il luogo di nascita nello studio longitudinale torinese. Epidemiologia e Prevenzione 1990;44:31-42.

24 Liberatos P, Link BG, Kelsey JC. The measurement of social class in epidemiology. Epidemiol Rev 1988;10:87121.

25 La Vecchia C, Pagano R, Negri E, Decarli A. Determinants of alcohol consumption in Italy. Int 7 Epidemiol 1987;16: 295.

26 Ferraroni M, La Vecchia C, Pagano R, Negri E, Decarli A. Smoking in Italy, 1986-1987. Tumori. 1989;75:521-26.

27 D'Amicis A, Faggiano F, Saba A. Distribuzione sociale dello stato nutrizionale in Italia negli anni ' 80 . In: Costa G, Faggiano F, eds. Rapporto sull'equità nella salute in Italia. Rome: Franco Angeli, 1994

28 Ferraroni M, La Vecchia C, Pagano R, Negri E, Parazzini C, Decarli A. Pattern of cervical screening utilization in Italy. Tumori, 1989;75:521-5.

29 Marmot MG, Morris JN. The social environment. In: Holland WW, Deteld R, Knox G, eds. Oxford textbook of public health. Vol. 1). Oxford: Oxford University Press, 1984.

health. Vol. 1). Oxford: Oxford University Press, 1984.
30 ISTAT. Annuario statistico Italiano. Roma: ISTAT, 1991. 